Although this volume deals with T-cell products and the collaboration of $\mathrm{T}$ and $\mathrm{B}$ cells, its distinctive contribution is on the preparation and properties of thymus hormones. At least some of these hormones are fairly stable as indicated by the use of heat to precipitate irrelevant protein in their preparation. Their properties include the ability to increase the number of $T$ cells, as judged by $E$ rosettes, when added to lymphocytes of patients with immune deficiency affecting $T$ cells, the ability to cause certain bone-marrow cells to acquire thymus surface-antigens, the ability to restore in-vitro graft-versus-host reactions to the spleen of irradiated, neonatally thymectomized and aged mice, the increase of antibody production in irradiated animals and the hastening of antibody production during the neonatal period.

The volume clearly puts thymus hormones on the map and raises the question of their use in man. Many problems, however, remain unsolved. First, is there a whole range of thymus hormones? There is some evidence that thymus hormone limits autoantibody production in NZB mice and may be needed for the maintenance of suppressor $T$ cells. There is other evidence that thymus hormone favours the restoration of antibody production and cell-mediated immunity after irradiation. The relatively slight effect of adult thymectomy in man suggests that there are several thymus hormones with opposing effects. It would be useful to know how many different hormones exist.

Second, most endocrine organs have a complex feedback control of secretion so as to respond to changes in the environment. We now need to know what signals control the release of thumus hormones by the thymus.

Third, it is not clear which thymus hormones act locally, on cells entering the thymus and coming in close contact with thymus epithelial cells, and which normally act systemically. The loss of suppressor cells early after adult thymectomy suggests that this may be one situation in which thymus hormones act systemically.

This book is excellent value for those interested in thymus hormones and should hasten the advance of knowledge of these factors and their application to human disease.

$$
\text { G. L. AsHERSON }
$$

\title{
Dermatophytes in human skin, hair and nails
}

By J. T. SInski. 1974. Springfield, Illinois, USA: Charles C. Thomas. Pp. xiv and 43; 35 figs. $\$ 7.95$.

This is quite a useful book on the identification of dermatophytes in clinical specimens. However, most of those who do this work will surely already have an " Atlas of medical mycology ", or indeed a general handbook of laboratory techniques, which probably covers the ground adequately.

H. I. WINNER 